\title{
Integrity monitoring for precise orbit determination of LEO satellites
}

\author{
Kan Wang ${ }^{1,2,3} \cdot$ Ahmed El-Mowafy $^{1}\left[\right.$ Chris Rizos $^{4}$
}

Received: 9 April 2021 / Accepted: 7 November 2021 / Published online: 24 December 2021

(c) The Author(s) 2021

\begin{abstract}
Due to an increasing requirement for high accuracy orbital information for low Earth orbit (LEO) satellites, precise orbit determination (POD) of LEO satellites is a topic of growing interest. To assure the safety and reliability of the applications requiring high accuracy LEO orbits in near-real-time, integrity monitoring (IM) is an essential operation of the POD process. In this contribution, the IM strategy for LEO POD in both the kinematic and reduced-dynamic modes is investigated. The overbounding parameters of the signal-in-space range error are investigated for the GPS products provided by the International GNSS Service's Real-Time Service and the Multi-GNSS Advanced Demonstration of Orbit and Clock Analysis service. Benefiting from the dynamic models used and the improved model strength, the test results based on the data of the LEO satellite GRACE FO-1 show that the average-case mean protection levels (PLs) can be reduced from about 3-4 m in the kinematic mode to about $1 \mathrm{~m}$ in the reduced-dynamic mode in the radial, along-track and cross-track directions. The overbounding mean values of the SISRE play the dominant role in the final PLs. In the reduced-dynamic mode and averagecase projection, the IM availabilities reach above $99 \%$ in the radial, along-track and cross-track directions with the alert limit (AL) set to $2 \mathrm{~m}$. The values are still above $98 \%$ with the AL set to $4 \mathrm{~m}$, when the duty cycle of tracking is reduced to $40 \%$, e.g., in the case of power limits for miniature satellites such as CubeSats.
\end{abstract}

Keywords LEO $\cdot$ POD $\cdot$ Integrity monitoring $\cdot$ GPS $\cdot$ Protection level

\section{Introduction}

Precise orbit determination (POD) of low Earth orbit (LEO) satellites has been intensively investigated in the past decades (Montenbruck et al. 2005; Gu et al. 2017). By applying the reduced-dynamic model integrated with GNSS observations tracked onboard and the use of comprehensive dynamic models, the accuracy of LEO POD can reach a few centimeters in "near-real-time," as a result of the availability of precise GNSS orbital and clock products provided by realtime services (Montenbruck et al. 2013; Allahvirdi-Zadeh et al. 2021). Such (near)-real-time LEO POD is required

Ahmed El-Mowafy

a.el-mowafy@curtin.edu.au

1 School of Earth and Planetary Sciences, Curtin University, Perth, Australia

2 National Time Service Center, Chinese Academy of Sciences, Xi' an, China

3 University of Chinese Academy of Sciences, Beijing, China

4 School of Civil and Environmental Engineering, UNSW, Sydney, Australia for applications such as Interferometric Synthetic Aperture Radar (InSAR) (Montenbruck 2017) and radio occultation analyses (Montenbruck et al. 2005). In recent years, LEO mega-constellations have been proposed as an augmentation to GNSS-based positioning, navigation and timing (PNT) services (Reid et al. 2018; Han et al. 2020). Compared to traditional GNSS-based positioning, LEO-aided GNSS positioning can benefit from significantly increased satellite numbers, improved satellite geometry, stronger signal strengths (Reid et al. 2018) and greater satellite velocities, which reduce the precise point positioning (PPP) convergence time ( $\mathrm{Ge}$ et al. 2018). As one of the conditions for LEO-aided positioning, the precise orbits of the LEO satellites are essential information.

Integrity monitoring (IM) in the near-real-time of the LEO satellite POD is important to ensure reliable positioning, thus ensuring safety and reliability for diverse user applications. With a pre-defined probability of misleading information (PMI), the protection level (PL) is a safety parameter that is computed to bound the orbital positioning errors (PEs). When the PL exceeds a pre-defined alert limit (AL), a warning message can be sent to the LEO system so 
that the affected satellite can be, e.g., marked as "unhealthy" and excluded from further processing. While LEO POD has been intensively studied, the IM of LEO POD is a new topic worthy of study.

IM has been investigated for different positioning methods for airborne and ground-based users in the last few decades. In aviation, the Advanced Receiver Autonomous Integrity Monitoring (ARAIM) algorithm (Blanch et al. 2012) was developed for dual-frequency multi-constellation (DFMC) signals to facilitate the vertical and horizontal guidance of aircraft. The algorithm was extended to ground-based applications using other positioning methods (El-Mowafy and Kubo 2018; Hassan et al. 2020). At the same time, the traditional single-frequency single-constellation satellite-based augmentation system (SBAS) is transitioning to a new generation that supports DFMC users (Wu et al. 2020). Its IM method is defined for aeronautical users (EUROCAE 2019), and extended for ground-based positioning of users for the more complex measurement environments encountered on the ground (Wang et al. 2021).

As a user of the GNSS signals in space, the IM of the dual-frequency LEO POD shares similar principles with the aeronautical users using dual-frequency GNSS signals. For instance, LEO satellites are flying under "open-sky" conditions and experience a relatively simple multipath environment. Furthermore, the first-order ionospheric delays are eliminated through the ionosphere-free (IF) combination. However, situations are also different compared to, e.g., those in aviation using the ARAIM or SBAS methods. The tropospheric residuals (after modeling) do not need to be considered in the IM anymore due to the altitude of the LEO satellites. Instead of the carrier-smoothed code observations used in the ARAIM and SBAS, the phase observations are directly used in LEO POD and hence influence the final POD precision and accuracy.

Near-real-time LEO POD relies on high accuracy realtime GNSS orbital and clock products generated by different providers. Service such as the International GNSS Service (IGS)'s Real-Time Service (RTS) (https://www.igs. org/rts/products/; El-Mowafy et al. 2017) is one example of ground-based processing that provides real-time POD. Services with corrections broadcast through GNSS or GEO satellites, e.g., the Multi-GNSS Advanced Demonstration of Orbit and Clock Analysis (MADOCA) service (Zhang et al. 2019) based on the Japanese Quasi-Zenith Satellite System (QZSS), and commercial services provided by, e.g., Fugro (Hauschild et al. 2016), also demonstrate possibilities for future onboard high accuracy POD. As the signalin-space range errors (SISRE) of these real-time products do not strictly follow a zero-mean normal distribution (Rife et al. 2006) as assumed in the POD least-squares processing, the overbounding parameters of the SISRE need to be determined using appropriate methods. The impact of different overbounding parameters on the LEO orbital PLs also requires investigation. Finally, LEO POD in the reduceddynamic mode involves the estimation of orbital dynamic parameters and the numerical integration of the orbits. As a result, numerically integrated partial derivatives of the Cartesian orbits with respect to the estimable orbital parameters are needed in the IM procedure for reduced-dynamic POD.

In this study, an IM procedure is proposed for LEO POD. The overbounding parameters are then investigated for the SISRE of real-time GNSS products under the average-case and worst-case for the orbital and clock error projection in the signal direction. Subsequently, the POD results and the PLs are assessed using real data from the GRACE FollowOn mission. The impacts of the overbounding parameters of the SISRE on the PLs are studied, and the PLs are investigated for miniature satellites for scenarios of non-continuous satellite tracking. The conclusions are then drawn and presented at the end.

\section{Processing strategy}

LEO POD and IM are performed for the reduced-dynamic mode, combining dynamic models with the GPS L1 and L2 phase observations. The code observations are used to pre-process the a priori clock synchronization (Dach et al. 2015). The IF combination is formed to remove the firstorder ionospheric delays so that only the orbital parameters, the LEO satellite clocks $\Delta \tilde{t}_{\mathrm{L}}$, and the IF float-valued phase ambiguities $n_{\mathrm{IF}}$ remain as estimable parameters in the linearized observation equations:

$\mathrm{E}\left(\Delta \varphi_{\mathrm{IF}}\right)=A_{\mathrm{K}} \Delta K+A_{\mathrm{D}} \Delta D+A_{\delta} \delta+c \times \Delta \tilde{t}_{\mathrm{L}}+\lambda_{\mathrm{IF}} n_{\mathrm{IF}}$

With

$\lambda_{\mathrm{IF}}=\frac{c}{f_{1}+f_{2}}$

$n_{\mathrm{IF}}=\frac{f_{1}^{2} \lambda_{1} n_{1}-f_{2}^{2} \lambda_{2} n_{2}}{c\left(f_{1}-f_{2}\right)}-\frac{\xi_{\mathrm{IF}}^{\mathrm{G}}-d_{\mathrm{IF}}^{\mathrm{G}}}{\lambda_{\mathrm{IF}}}-n_{\mathrm{IF}}^{0}$

$\Delta \tilde{t}_{\mathrm{L}}=\Delta t_{\mathrm{L}}+\frac{\xi_{\mathrm{L}, \mathrm{IF}}+\lambda_{\mathrm{IF}} n_{\mathrm{IF}}^{0}}{c}$

where $\mathrm{E}(\bullet)$ is the expectation operator, and $\Delta \varphi_{\mathrm{IF}}$ denotes the observed-minus-computed (O-C) terms of the IF phase observations. $c$ denotes the speed of light, and $f_{j}, n_{j}, \lambda_{j}$ $(j=1,2)$ denotes the frequency, ambiguity and wavelength on frequency $j$, respectively. As the absolute values of LEO satellite clocks and the IF float ambiguities are not of concern in this study, a reference ambiguity $n_{\mathrm{IF}}^{0}$ (with the value of zero) was set to avoid the rank deficiency between the 
LEO satellite clocks and the ambiguities. The terms $\xi_{\mathrm{IF}}^{\mathrm{G}}$ and $d_{\mathrm{IF}}^{\mathrm{G}}$ denote the GPS IF satellite phase and code bias vector, respectively, and $\xi_{\mathrm{L}, \mathrm{IF}}$ refers to the LEO IF satellite phase bias. $\Delta K$ and $\Delta D$ are the increment vectors of the six Keplerian elements at the initial state and the time-constant orbital dynamic parameters with respect to the a priori values, respectively. $\delta$ denotes the vector of the stochastic piece-wise constant accelerations. $\Delta D$ and $\delta$ are given in the radial (R), along-track (S) and cross-track (W) directions with the stochastic accelerations $\delta$ estimated, in this study, within 6 min time intervals and constrained to zeros with a standard deviation $\left(\sigma_{\delta}\right)$ of $5 \times 10^{-6}\left[\mathrm{~s}^{-2}\right] \times \sigma_{0}$. The a priori standard deviation of unit weight $\left(\sigma_{0}\right)$ refers to the zenith direction on L1. $A_{\mathrm{K}}, A_{\mathrm{D}}$ and $A_{\delta}$ contain the partial derivatives of the observations with respect to the Keplerian elements, the dynamic parameters and the stochastic accelerations, respectively, which can be further expressed as:

$A_{\mathrm{K}}=\frac{\partial \varphi_{\mathrm{IF}}}{\partial r_{\mathrm{I}}} \frac{\partial r_{\mathrm{I}}}{\partial K}$

$A_{\mathrm{D}}=\frac{\partial \varphi_{\mathrm{IF}}}{\partial r_{\mathrm{I}}} \frac{\partial r_{\mathrm{I}}}{\partial D}$

$A_{\delta}=\frac{\partial \varphi_{\mathrm{IF}}}{\partial r_{\mathrm{I}}} \frac{\partial r_{\mathrm{I}}}{\partial \delta}$

where $\partial \varphi_{\mathrm{IF}} / \partial r_{\mathrm{I}}$ denotes the partial derivatives of the observation with respect to the LEO position vector in the inertial system, i.e., the GPS-to-LEO unit direction vectors. $\partial r_{\mathrm{I}} / \partial K$ and $\partial r_{\mathrm{I}} / \partial D$ are the partial derivatives of the LEO position vector with respect to the Keplerian elements and the dynamic parameters, respectively. These partial derivatives are numerically integrated based on the variational equations (Montenbruck and Gill 2000). $\partial r_{\mathrm{I}} / \partial \delta$, the partial derivatives of the position vector with respect to the stochastic accelerations, can be computed as linear combinations of $\partial r_{\mathrm{I}} / \partial K$ (Dach et al. 2015).

The unknowns, contained in the increment vector $\Delta x$ containing all the orbital dynamic parameters, the LEO satellite clock parameters of all epochs and the ambiguity parameters of all epochs and all satellites, are estimated in a least-squares adjustment. The variance-covariance $Q_{l}$ of all the observations can be formulated as a block diagonal matrix constructed from the variance-covariance matrices of the phase O-C terms at each time point $t_{i}$, denoted as $Q_{\Delta \varphi}\left(t_{i}\right)$, and the variance matrix of the constraints for stochastic accelerations:

$Q_{l}=\operatorname{blkdiag}\left(Q_{\Delta \varphi}\left(t_{1}\right), \cdots, Q_{\Delta \varphi}\left(t_{n}\right), \sigma_{\delta}^{2} \times I_{p}\right)$

where the operator blkdiag $(\bullet)$ forms the block diagonal matrix. For epoch $t_{i}$, the variance-covariance matrix of the
Table 1 Dynamic models used for the LEO POD (JPL refers to NASA's Jet Propulsion Laboratory. IERS is the abbreviation for international Earth Rotation and Reference Systems Service)

\begin{tabular}{ll}
\hline Item & Model \\
\hline Earth's gravity field & $\begin{array}{c}\text { EGM2008 (degree: 120) (Pavlis et al. } \\
\text { 2008) }\end{array}$ \\
Gravity of other planets & $\begin{array}{c}\text { Ephemeris from JPL DE405 (Standish } \\
\text { 1998) }\end{array}$ \\
Solid earth and pole tides & IERS Conventions 2010 (Petit and Luzum \\
2010) \\
Ocean tides & FES2004 (Lyard et al. 2006) \\
\hline
\end{tabular}

phase IF O-C terms is expressed as a diagonal matrix of the corresponding variances for each satellite $s(1, \cdots, m)$, with $m$ denoting the number of satellites:

$Q_{\Delta \varphi}\left(t_{i}\right)=\operatorname{diag}\left(\sigma_{1}^{2}\left(t_{i}\right), \cdots, \sigma_{m}^{2}\left(t_{i}\right)\right)$

In this study, as was done for Sentinel satellite POD products and in the Bernese LEO campaign, equal weighting is applied for all the phase IF O-C terms with $\sigma_{s}^{2}=\left(f_{1}^{4}+f_{2}^{4}\right) /\left(f_{1}^{2}-f_{2}^{2}\right)^{2} \times \sigma_{0}^{2}$, as the GNSS receiver onboard LEO satellite experiences less elevation-dependency for the noise and multipath effects than on the ground, so that traditional elevation-dependent weighting models might not lead to better results.

For LEO POD, the least-squares adjustment is performed using $24 \mathrm{~h}$ of GPS observations with a sampling interval of $30 \mathrm{~s}$, which is sufficient for LEO POD (Wang et al. 2020). The dynamic models used for numerical integration are summarized in Table 1. The LEO orbits are numerically integrated in the inertial system with all the orbital parameters solved in a least-squares adjustment.

In addition to the reduced-dynamic mode, for comparison purposes, the POD is also performed in the kinematic mode (i.e., without using any dynamic model). In such a case, the orbits are directly estimated in the form of the Cartesian coordinates of the satellite in the Earth-fixed system.

\section{Integrity monitoring}

For the IM, the variance-covariance matrix of the observations is constructed assuming Gaussian noise that overbounds all existing errors remaining in the $\mathrm{O}-\mathrm{C}$ terms. The variance-covariance $\tilde{Q}_{l}$ of the observations for the purpose of integrity can be formulated as:

$\tilde{Q}_{l}=\operatorname{blkdiag}\left(\tilde{Q}_{\Delta \varphi}\left(t_{1}\right), \cdots, \tilde{Q}_{\Delta \varphi}\left(t_{n}\right), \tilde{\sigma}_{\delta}^{2} \times I_{p}\right)$

With

$\tilde{Q}_{\Delta \varphi}\left(t_{i}\right)=\operatorname{diag}\left(\tilde{\sigma}_{1}^{2}\left(t_{i}\right), \cdots, \tilde{\sigma}_{m}^{2}\left(t_{i}\right)\right)$ 
Taking into account the factors considered for IM in aviation using the DFMC SBAS (EUROCAE 2019) that also operates in an open-sky measurement environment and uses undifferenced IF observations, the variance element $\tilde{\sigma}_{s}^{2}$ $(s=1, \cdots, m)$ can be defined considering the variance of the noise and multipath $\left(\tilde{\sigma}_{\text {air }}^{2}\right)$, the variance of the GNSS orbital and clock errors projected into the signal direction $\left(\tilde{\sigma}_{\text {GNSS }}^{2}\right)$ and the variance of the ionospheric residuals after forming the IF cothe overbounding standardmbination $\left(\tilde{\sigma}_{\text {iono }}^{2}\right)$, which is assumed to account for miss-modeled ionospheric residuals that will be discussed later:

$\tilde{\sigma}_{s}^{2}=\tilde{\sigma}_{\text {air }}^{2}+\tilde{\sigma}_{\text {GNSS }}^{2}+\tilde{\sigma}_{\text {iono }}^{2}$

Note that LEO satellites are above the troposphere, and thus, there are no tropospheric delays to consider. The variance $\sigma_{\text {air }}^{2}$ is modeled with the overbounding standard deviations of the single-frequency noise $\left(\sigma_{\mathrm{N}}\right)$ of geodetic-grade antennas and the multipath $\left(\sigma_{\mathrm{M}}\right)$ under open-sky conditions as: $\tilde{\sigma}_{s}$ in the zenith direction divided by the IF amplification factor $\sqrt{f_{1}^{4}+f_{2}^{4}} /\left(f_{1}^{2}-f_{2}^{2}\right)$.

After computing $\tilde{Q}_{l}$ from (10), the variance-covariance matrix $\tilde{Q}_{\Delta x}$ of the unknown vector is:

$\tilde{Q}_{\Delta x}=\left(A^{\mathrm{T}} \tilde{Q}_{l}^{-1} A\right)^{-1}$

where $A$ is the total design matrix (including the part of the absolute constraint) for the reduced-dynamic POD. Next, the variance-covariance matrix of the orbital parameters denoted as $\tilde{Q}_{\mathrm{O}}$, is derived from $\tilde{Q}_{\Delta x}$ as the first $729 \times 729$ elements in the reduced-dynamic mode, accounting for six Keplerian elements at the initial condition, one set of 3D time-constant dynamic parameters, and 240 sets of 3D piece-wise constant stochastic accelerations (within 6 min intervals). The variance-covariance matrix of the orbital parameters $\tilde{Q}_{\mathrm{O}}$ obtained through the least-squares adjustment is transformed to that of the Cartesian coordinates in the

$\tilde{\sigma}_{\text {air }}^{2}=\frac{f_{1}^{4}+f_{2}^{4}}{\left(f_{1}^{2}-f_{2}^{2}\right)^{2}} \times\left(\sigma_{\mathrm{N}}^{2}+\sigma_{\mathrm{M}}^{2}\right)=\frac{f_{1}^{4}+f_{2}^{4}}{\left(f_{1}^{2}-f_{2}^{2}\right)^{2}} \times \frac{\left(f_{1}^{2}-f_{5}^{2}\right)^{2}}{f_{1}^{4}+f_{5}^{4}} \times \frac{\tilde{\sigma}_{\mathrm{N}, \mathrm{DFMC}}^{2}+\tilde{\sigma}_{\mathrm{M}, \mathrm{DFMC}}^{2}}{\beta^{2}}$

where $\tilde{\sigma}_{\mathrm{N}, \mathrm{DFMC}}$ and $\tilde{\sigma}_{\mathrm{M}, \mathrm{DFMC}}$ are the overbounding standard deviations of the IF carrier-smoothed observation noise and multipath after 100 epochs using L1 and L5, applied for aeronautical users with the DFMC SBAS as defined in EUROCAE (2019). The factor $\beta$ (13) is set to 10, assuming the standard deviation of the raw code noise and multipath is 100 times that of the phase noise and multipath, and the code-smoothing over 100 epochs reduces this amplification factor to 10 . The calculation of $\tilde{\sigma}_{\text {iono }}$ follows that for the case of DFMC SBAS, i.e., an elevation-angle dependent function accounting for the high-order ionospheric delays, ray bending and excess Total Electron Content (TEC). The ionosphere differently influences the LEO satellites at different altitudes. Some of them could even be above the ionosphere; therefore, we made a conservative assumption using the same formula as for the aircraft. The overbounding standard deviation for the orbital and clock errors projected into the signal direction $\left(\tilde{\sigma}_{\mathrm{GNSS}}\right)$ is dependent on the service provider of the real-time GNSS products.

Recall that stochastic accelerations are constrained to zero with the standard deviation $\sigma_{\delta}=5 \times 10^{-6}\left[\mathrm{~s}^{-2}\right] \times \sigma_{0}$. To keep the same weight of constraint for the stochastic accelerations as that in the POD, the standard deviation for the constraint $\left(\tilde{\sigma}_{\delta}^{2} \mathrm{in} \mathrm{m} / \mathrm{s}^{2}\right)$ for integrity purposes is expressed as:

$\tilde{\sigma}_{\delta}=5 \times 10^{-6}\left[s^{-2}\right] \times \tilde{\sigma}_{0}$

where $\tilde{\sigma}_{0}$ denotes the overbounding standard deviation of unit weight on L1 (in the unit of meters), calculated with the radial, along-track and cross-track directions at each epoch using the error propagation law, such that:

$\tilde{Q}_{\mathrm{RSW}}\left(t_{i}\right)=F\left(t_{i}\right) \tilde{Q}_{\mathrm{O}} F^{\mathrm{T}}\left(t_{i}\right)$

with the transformation matrix $F\left(t_{i}\right)$ containing the partial derivatives of the RSW coordinates with respect to the orbital parameters expressed as:

$F\left(t_{i}\right)=R_{\mathrm{I} 2 \mathrm{~T}}\left(t_{i}\right) \frac{\partial r_{\mathrm{I}}\left(t_{i}\right)}{\partial O}$

where $\partial r_{\mathrm{I}}\left(t_{i}\right) / \partial O$ represents the partial derivatives of the position vector in the inertial system at $t_{i}$ with respect to all the orbital parameters $O$, which need to be computed by numerical integration based on the variational equations and their linear combinations (as mentioned earlier). $R_{\mathrm{I} 2 \mathrm{~T}}\left(t_{i}\right)$ denotes the rotation matrix from the inertial system to the RSW-system at $t_{i}$. In this study, $R_{\mathrm{I} 2 \mathrm{~T}}$ at $t_{i}$ is approximated by:

$R_{\mathrm{I} 2 \mathrm{~T}}=\left[\frac{r_{\mathrm{I}}}{\left\|r_{\mathrm{I}}\right\|}, \frac{v_{\mathrm{I}}}{\left\|v_{\mathrm{I}}\right\|}, \frac{r_{\mathrm{I}} \times v_{\mathrm{I}}}{\left\|r_{\mathrm{I}} \times v_{\mathrm{I}}\right\|}\right]^{\mathrm{T}}$

where $r_{\mathrm{I}}$ and $v_{\mathrm{I}}$ represents the LEO position and velocity vectors, respectively, and $\|\cdot\|$ is the norm of the vector.

For IM, the PL is computed covering the positional errors with a pre-defined PMI (Shively 2014) as mentioned in the Introduction. Assuming that the PMI is equally distributed in the three directions, the PLs of the orbital element $q$ can then be formulated as: 
$\mathrm{PL}_{q}\left(t_{i}\right)=K_{q} \times \tilde{\sigma}_{\mathrm{RSW}, q}\left(t_{i}\right)+\left|S_{\mathrm{RSW}, q}\left(t_{i}\right)\right| \times \tilde{b}, q=1,2,3$

with

$K_{q}=C^{-1}\left(1-\frac{\mathrm{PMI}}{3 \times 2}\right)$

$S_{\mathrm{RSW}, q}\left(t_{i}\right)=e_{q}^{\mathrm{T}} F\left(t_{i}\right) F_{\mathrm{O}} \tilde{Q}_{\Delta x} A^{\mathrm{T}} \tilde{Q}_{l}^{-1}$

$\tilde{b}=\left[\tilde{m}_{\mathrm{GNSS}} \times J_{l_{\Delta \varphi}, 0}\right]^{\mathrm{T}}$

where $\mathrm{C}^{-1}($.$) is the inverse CDF of the standard normal dis-$ tribution, $\tilde{\sigma}_{\mathrm{RSW}, q}\left(t_{i}\right)$ is the overbounding standard deviation of the $q$-th orbital element at epoch $t_{i}$, with $q=1,2,3$ representing the orbital component in the radial, along-track and cross-track directions, respectively, which can be derived from $\tilde{Q}_{\mathrm{RSW}}$ in Eq. 16. $\tilde{m}_{\mathrm{GNSS}}$ denotes here the scalar overbounding mean value of the GNSS orbital and clock error projections that are computed along with the $\tilde{\sigma}_{\mathrm{GNSS}}$ (Eq. 12) (which will be explained in the next section). $J_{l_{\Delta \varphi}}$ is a vector of ones with length $l_{\Delta \varphi}$, which denotes the number of used phase IF observations. $S_{\mathrm{RSW}, q}$ projects the bias vector $\tilde{b}$ from the observation to the position domain at the corresponding epoch. The matrix $F_{\mathrm{O}}$ selects the orbital parameters from the entire unknown vector containing the orbital parameters, the LEO satellite clock parameters and the float ambiguity parameters (see Eq. 1), and $e_{q}$ is a $3 \times 1$ vector with the $q$-th element set to the value of one and the other elements set to zeros.

In the proposed algorithm, large cycle slips or biases will be detected by the fault detection and exclusion (FDE) step. Concerning the remaining smaller biases that could vary over time, compared to the ambiguity-fixed solutions, the float ambiguities could absorb satellite-specific errors that vary little with time. The values of the float ambiguities themselves are not of concern. Other small miss-detected biases errors could remain in the observation residuals considered in $\tilde{b}$ (Eq. 19), which represents possible nominal biases under the system normal operation, and their random variabilities could be considered in the stochastic term $\tilde{\sigma}_{s}^{2}$ (Eq. 12). These parameters can be properly set based on a future comprehensive study.

Compared to the reduced-dynamic mode, the procedure for the IM in the kinematic mode is simpler, as the variance-covariance matrix of the Cartesian orbits can be directly obtained within the least-squares adjustment as:

$\tilde{Q}_{\Delta x_{k}}=\left(A_{k}^{\mathrm{T}} \tilde{Q}_{\Delta \varphi}^{-1} A_{k}\right)^{-1}$

where the design matrix $A_{\mathrm{k}}$ can be expressed with the help of the combined GNSS-to-LEO unit direction vectors for all observations $\left(A_{\mathrm{I}, n}\right)$, the design matrix for the LEO clock parameters $A_{T, n}$, as well as that for the ambiguity vector $\left(\Lambda_{\mathrm{IF}}\right)$ as:

$A_{k}=\left[A_{\mathrm{I}, n} A_{T, n} \Lambda_{\mathrm{IF}}\right]$

The variance-covariance matrix of the orbits $\tilde{Q}_{r_{\mathrm{I}}}\left(t_{i}\right)$ $(i=1, \cdots, n)$ are contained in the first $3 n \times 3 n$ elements of $\tilde{Q}_{\Delta x_{\mathrm{k}}}$. To obtain the variance-covariance matrix of the orbits in the radial, along-track and cross-track directions, i.e., $\tilde{Q}_{\mathrm{RSW}, \mathrm{k}}\left(t_{i}\right)$, similar to the reduced-dynamic mode, transformation is required from the inertial system to the RSW-system:

$\tilde{Q}_{\mathrm{RSW}, k}\left(t_{i}\right)=R_{\mathrm{I} 2 \mathrm{~T}}\left(t_{i}\right) \tilde{Q}_{r_{\mathrm{I}}}\left(t_{i}\right) R_{\mathrm{I} 2 \mathrm{~T}}^{\mathrm{T}}\left(t_{i}\right)$

with the overbounding standard deviation of the orbits obtained in the radial, along-track and cross-track directions $\left(\tilde{\sigma}_{\mathrm{RSW}, \mathrm{k}, q}\right)$, the corresponding protection levels in the kinematic mode $\left(\mathrm{PL}_{\mathrm{k}, q}\right)$ can be computed:

$$
\begin{aligned}
\mathrm{PL}_{k, q}\left(t_{i}\right)= & \mathrm{C}^{-1}\left(1-\frac{\mathrm{PMI}}{3 \times 2}\right) \times \tilde{\sigma}_{\mathrm{RSW}, k, q}\left(t_{i}\right) \\
& +\left|S_{\mathrm{RSW}, q}\left(t_{i}\right)\right| \times \tilde{b}_{k}, q=1,2,3
\end{aligned}
$$

with

$S_{\mathrm{RSW}, k, q}\left(t_{i}\right)=e_{q}^{\mathrm{T}} R_{\mathrm{I} 2 \mathrm{~T}}\left(t_{i}\right) F_{k}\left(t_{i}\right) \tilde{Q}_{\Delta x_{k}} A_{k}^{\mathrm{T}} \tilde{Q}_{\Delta \varphi}^{-1}$

$\tilde{b}_{k}=\tilde{m}_{\mathrm{GNSS}} \times J_{l_{\Delta \varphi}}$

where $F_{\mathrm{k}}$ selects the kinematic orbital positions of the corresponding epoch from the entire vector of unknowns. In summary, compared to the existing IM algorithm for the DFMC SBAS, the LEO IM are subject to the following changes:

- The reduced-dynamic LEO POD requires a more complicated scheme for the projection from the observation domain to the position domain as, instead of directly computing the Cartesian coordinates, the dynamic orbital parameters are estimated. In this regard, the objective of the least-squares adjustment in the reduced-dynamic POD is different from that of DFMC SBAS positioning. In the reduced-dynamic POD, the GNSS observations are used to improve the dynamic parameters in a leastsquares adjustment so that the final orbits are obtained through numerical integration using an improved dynamic model.

- Concerning the real-time GNSS satellite orbits and clocks, the LEO POD has more options, which may exhibit higher accuracy than using SBAS corrections.

- The model for LEO POD is stronger than that used by ground-based or airborne users, as LEO satellites are above the troposphere (i.e., it uses less term when com- 
Table 2 Contributions of different errors to the overbounding standard deviations and mean values of the projected GPS orbital and clock errors during the test period September 2018 with an average-case assumption

\begin{tabular}{|c|c|c|c|c|c|c|}
\hline Service & $\alpha_{\mathrm{R}} \times \tilde{\sigma}_{\mathrm{R}}[\mathrm{m}]$ & $c \times \tilde{\sigma}_{\Delta t_{\mathrm{G}}}[\mathrm{m}]$ & $\tilde{\sigma}_{\mathrm{R} \Delta t_{\mathrm{G}}}[\mathrm{m}]$ & $\alpha_{\mathrm{SW}} \times \tilde{\sigma}_{\mathrm{S}}[\mathrm{m}]$ & $\alpha_{\mathrm{SW}} \times \tilde{\sigma}_{\mathrm{W}}[\mathrm{m}]$ & $\tilde{\sigma}_{\mathrm{GNSS}}[\mathrm{m}]$ \\
\hline IGS RTS & 0.034 & 0.095 & 0.092 & 0.011 & 0.005 & 0.093 \\
\hline \multirow[t]{2}{*}{ MADOCA } & 0.056 & 0.065 & 0.066 & 0.009 & 0.007 & 0.067 \\
\hline & $\alpha_{\mathrm{R}} \times \tilde{m}_{\mathrm{R}}[\mathrm{m}]$ & $c \times \tilde{m}_{\Delta t_{\mathrm{G}}}[\mathrm{m}]$ & $\tilde{m}_{\mathrm{R} \Delta t_{\mathrm{G}}}[\mathrm{m}]$ & $\alpha_{\mathrm{SW}} \times \tilde{m}_{\mathrm{S}}[\mathrm{m}]$ & $\alpha_{\mathrm{SW}} \times \tilde{m}_{\mathrm{W}}[\mathrm{m}]$ & $\begin{array}{l}\tilde{m}_{\mathrm{GNSS}} \\
{[\mathrm{m}]}\end{array}$ \\
\hline IGS RTS & 0.016 & 0.047 & 0.034 & 0.008 & 0.002 & 0.043 \\
\hline MADOCA & 0.041 & 0.054 & 0.030 & 0.007 & 0.009 & 0.046 \\
\hline
\end{tabular}

puting the overbounding standard deviations for the observations).

- LEO POD uses directly the phase observations instead of the carrier-smoothed code observations as in DFMC SBAS. This ensures higher precision reduced-dynamic POD.

- In LEO POD, an overbounding bias term in the computation of the PLs is considered, which is not the case in the DFMC SBAS.

\section{SISRE analysis of real-time GNSS services}

As mentioned in the previous section, the overbounding standard deviation $\left(\tilde{\sigma}_{\mathrm{GNSS}}\right)$ and mean values $\left(\tilde{m}_{\mathrm{GNSS}}\right)$ of the GNSS orbital and clock errors, projected into the signal direction, are important components in the computation of PLs. In this study, two different cases are considered: the average-case and the worst-case.

\section{Average-case projection}

In the average-case, the SISRE assumes a uniform distribution of the points over the sphere of the approximate LEO orbital radius, with a projection factor $\alpha_{\mathrm{R}}$ defined for the radial direction, and a factor $\alpha_{\mathrm{SW}}$ for the along-track and cross-track directions, respectively (Heng et al. 2011). The overbounding standard deviation can thus be expressed as:

$\tilde{\sigma}_{\mathrm{GNSS}}=\sqrt{\tilde{\sigma}_{\mathrm{R} \Delta t_{\mathrm{G}}}^{2}+\alpha_{\mathrm{SW}}^{2} \times\left(\tilde{\sigma}_{\mathrm{S}}^{2}+\tilde{\sigma}_{\mathrm{W}}^{2}\right)}$

where $\tilde{\sigma}_{\mathrm{R} \Delta t_{\mathrm{G}}}$ is the overbounding standard deviation of the term $\left(\alpha_{\mathrm{R}} \times \Delta r_{\mathrm{R}}-c \times \Delta t_{\mathrm{G}}\right)$ considering the correlation between the radial orbital error $\Delta r_{R}$ and the satellite clock error $\Delta t_{\mathrm{G}}$ of the GNSS satellites. $\tilde{\sigma}_{\mathrm{S}}$ and $\tilde{\sigma}_{\mathrm{W}}$ represent the overbounding standard deviations of the along-track and cross-track orbital errors of the GNSS satellites, respectively.

The overbounding mean value of the projected GNSS orbital and clock errors can be expressed as:
$\tilde{m}_{\mathrm{GNSS}}=\tilde{m}_{\mathrm{R} \Delta t_{\mathrm{G}}}+\alpha_{\mathrm{SW}} \times\left(\tilde{m}_{\mathrm{S}}+\tilde{m}_{\mathrm{W}}\right)$

where $\tilde{m}_{\mathrm{R} \Delta t_{\mathrm{G}}}$ is the overbounding mean value of the $\operatorname{term}\left(\alpha_{\mathrm{R}} \times \Delta r_{\mathrm{R}}-c \times \Delta t_{\mathrm{G}}\right)$, and $\tilde{m}_{\mathrm{S}}$ and $\tilde{m}_{\mathrm{W}}$ refer to those of the along-track and cross-track orbital errors of the GNSS satellites, respectively. The factors $\alpha_{\mathrm{R}}$ and $\alpha_{\mathrm{SW}}$ are related to both the orbital heights of the LEO satellite and the GNSS satellites and do not vary much for LEO orbital altitudes from 500 to $1000 \mathrm{~km}$. The radial orbital error and the clock error play the dominant role in the overbounding standard deviation and mean values.

In this study, the overbounding standard deviation $\tilde{\sigma}_{\mathrm{GNSS}}$ and mean values $\tilde{m}_{\mathrm{GNSS}}$ are computed for two types of realtime GPS products, i.e., those from the IGS RTS and the MADOCA Service. The IGS final orbits and clocks are used as the reference for the assessment of the errors. Note that the real-time GPS satellite clocks are re-referenced to those of the IGS final clocks. The satellite-specific timeconstant mean values are removed from $\Delta t_{\mathrm{G}}$, as they can be absorbed by the float ambiguities (Zhang et al. 2019). The errors (all the single components and the radial-clock combined component) exceeding $0.5 \mathrm{~m}$ are excluded from the analysis, and the remaining errors exceeding $m_{\text {err }} \pm 10 \times \sigma_{\text {err }}$ are further screened as outliers, where $m_{\text {err }}$ and $\sigma_{\text {err }}$ denote the mean value and standard deviation of the corresponding error type, respectively. These outliers are also screened in the phase-preprocessing during the POD process.

A two-step overbounding strategy is used for the computation of the overbounding standard deviations and mean values (Blanch et al. 2018). Compared to the paired overbounding method (Rife et al. 2006), the two-step strategy has the advantage of generating a smaller overbounding mean value, which is essential for reducing the LEO PLs, and thus may improve solution availability, as will be explained in Section "Test results." In this study, no excess mass is set for the overbounding to avoid the high inflation of the actual probability of the overbounding distribution due to a large number of observations in the batch leastsquares POD process. 
Fig. 1 Average-case (left) and the worst-case (right) of the orbital contribution in SISRE for LEO satellites

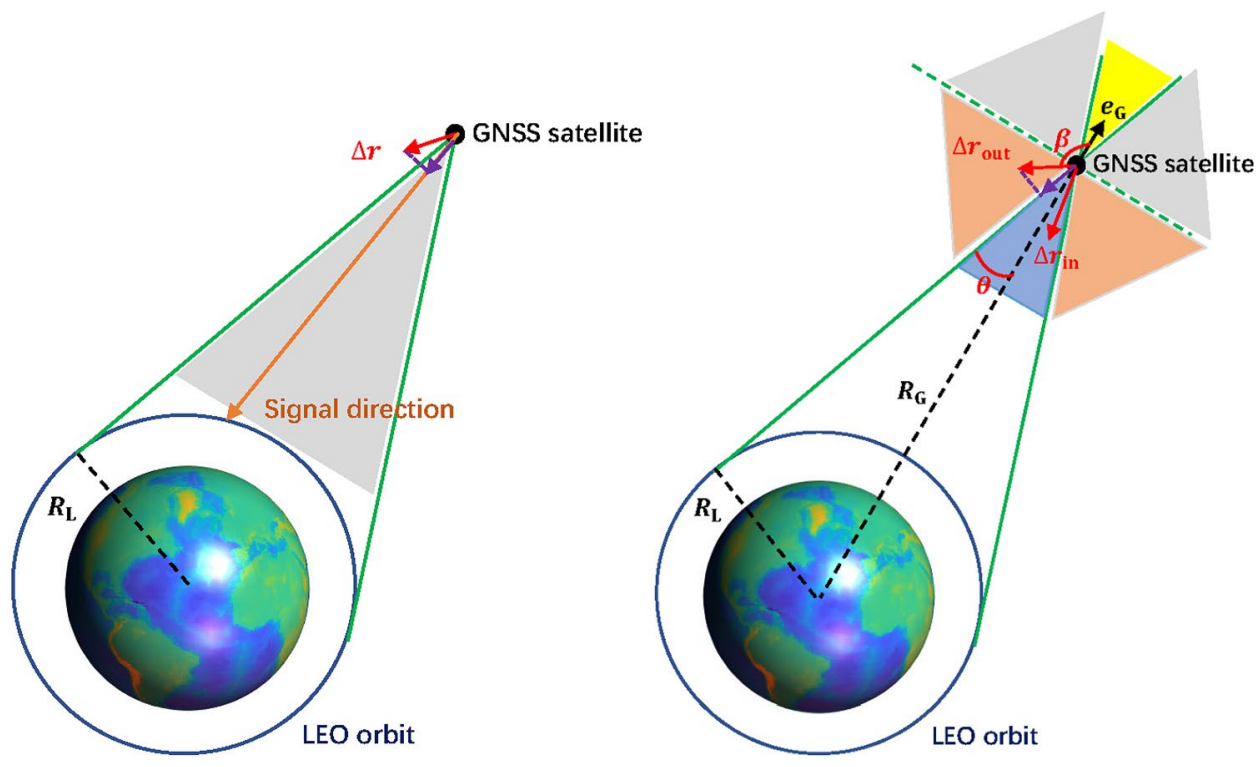

Table 2 lists the contributions of different errors to the overbounding standard deviation $\tilde{\sigma}_{\mathrm{GNSS}}$ and mean value $\tilde{m}_{\mathrm{GNSS}}$ for the orbital and clock projection errors assuming the average-case over the period September 2018. The LEO GRACE FO-1 satellite, with approximately $503 \mathrm{~km}$ orbital height (Wen et al. 2019), for the period from August 14 to 20, 2018 was used as an example. To show the correlation between the terms $\left(\alpha_{\mathrm{R}} \times \Delta r_{\mathrm{R}}\right)$ and $\left(c \times \Delta t_{\mathrm{G}}\right)$, the contributions are also given for the corresponding single terms with $\tilde{\sigma}_{\mathrm{R}}$ and $\tilde{m}_{\mathrm{R}}$ denoting the overbounding standard deviation and mean value of the radial orbital error, and $\tilde{\sigma}_{\Delta t_{\mathrm{G}}}$ and $\tilde{m}_{\Delta t_{\mathrm{G}}}$ denoting those of the clock error. For the purpose of comparison, all epochs having valid components are used for the analysis.

From Table 2 it can be seen that $\tilde{\sigma}_{\mathrm{R} \Delta t_{\mathrm{G}}}$ is smaller than the case when treating the radial orbital errors and the clocks as independent terms, which confirms the correlation between the two parameters. The situation is similar for the overbounding mean values $\tilde{m}_{\mathrm{R} \Delta t_{\mathrm{G}}}$. The $\tilde{m}_{\mathrm{GNSS}}$ of both products are within $5 \mathrm{~cm}$, and their $\tilde{\sigma}_{\mathrm{GNSS}}$ are at the sub-dm to dm-level.

\section{Worst-case projection}

The worst-case projection assumes the largest projection of the orbital and clock errors. Based on Montenbruck et al. (2015), the worst-case for the orbital contribution in SISRE is expanded from the case for ground-based users to the case for LEO satellites as shown in Fig. 1.

As the LEO orbits are near-circular, they are assumed to be circles with a radius $R_{\mathrm{L}}$. When the orbital error vector $\Delta r$ intersects with the sphere of radius $R_{\mathrm{L}}$ (see $\Delta r_{\text {in }}$ in the right panel of Fig. 1), the worst-case orbital error $\Delta \tilde{r}$ is assumed to have the absolute value of $\|\Delta r\|$ :
$\Delta \tilde{r}=\|\Delta r\|$, for the yellow area with $\beta \leq \theta$

$\Delta \tilde{r}=\|\Delta r\|$, for the blue area with $\beta \leq \pi-\theta$

With

$\beta=\arccos \left(\frac{\left\langle\Delta r, e_{\mathrm{G}}\right\rangle}{\|\Delta r\|}\right)=\arccos \left(\frac{\Delta r_{\mathrm{R}}}{\|\Delta r\|}\right)$

$\theta=\arcsin \left(\frac{R_{\mathrm{L}}}{R_{\mathrm{G}}}\right)$

where $<\bullet>$ is the dot product of two vectors, and $R_{\mathrm{L}}$ and $R_{\mathrm{G}}$ are approximated by the mean LEO orbital height and the mean GPS orbital height of $20,200 \mathrm{~km}$ (with the addition of the Earth radius of $6371 \mathrm{~km}$ as used in the previous subsection). $e_{\mathrm{G}}$ denotes the unit direction vector from the Earth to the GPS satellite, and $\Delta r_{\mathrm{R}}$ refers to the radial component of $\Delta r$. In the case when the orbital error vector does not intersect the sphere of radius $R_{\mathrm{L}}$ (see $\Delta r_{\text {out }}$ in the right panel of Fig. 1), the orbital error vector $\Delta r$ is projected into the nearest tangent line from the GNSS satellite to the sphere for LEO orbits using the relations:

$\Delta \tilde{r}=\|\Delta r\| \times \cos (\beta-\theta)$, for the grey area with, $\theta<\beta \leq \frac{\pi}{2}$

$\Delta \tilde{r}=\|\Delta r\| \times \cos (\beta+\theta)$, for the orange area with, $\frac{\pi}{2}<\beta \leq \pi-\theta$

As the worst-case orbital contributions to SISRE might not be normally distributed, similar to the case in the previous section, an overbounding standard deviation $\left(\tilde{\sigma}_{\Delta \tilde{r}}\right)$ and mean value $\left(\tilde{m}_{\Delta \tilde{r}}\right)$ need to be determined for $\Delta \tilde{r}$. As a pessimistic 
Table 3 Overbounding standard deviations and mean values of the GPS orbital and clock errors during the test period September 2018 under the worst-case assumption

\begin{tabular}{lllll}
\hline Service & $\tilde{\sigma}_{\Delta \tilde{r}}[\mathrm{~m}]$ & $c \times \tilde{\sigma}_{\Delta t_{\mathrm{G}}}[\mathrm{m}]$ & $\tilde{\sigma}_{\Delta \tilde{r} \Delta t_{\mathrm{G}}}[\mathrm{m}]$ & 0.095 \\
\hline IGS RTS & 0.045 & 0.096 & $\tilde{\sigma}_{\mathrm{GNSS}}[\mathrm{m}]$ \\
\hline MADOCA & 0.073 & 0.072 & 0.071 & 0.106 \\
\hline IGS RTS & $\tilde{m}_{\Delta \tilde{r}}[\mathrm{~m}]$ & $c \times \tilde{m}_{\Delta t_{\mathrm{G}}}[\mathrm{m}]$ & $\tilde{m}_{\Delta \tilde{r} \Delta t_{\mathrm{G}}}[\mathrm{m}]$ & 0.102 \\
MADOCA & 0.026 & 0.043 & 0.027 & 0.070 \\
\hline
\end{tabular}

assumption, without considering the correlation between the radial orbits and the satellite clock errors (Montenbruck et al. 2015), the overbounding standard deviation and mean value of the projected GNSS orbital and clock errors can be expressed as:

$\tilde{\sigma}_{\mathrm{GNSS}}=\sqrt{\tilde{\sigma}_{\Delta \tilde{r}}^{2}+c^{2} \times \tilde{\sigma}_{\Delta t_{\mathrm{G}}}^{2}}$

$\tilde{m}_{\mathrm{GNSS}}=\tilde{m}_{\Delta \tilde{r}}+c \times \tilde{m}_{\Delta t_{\mathrm{G}}}$

Using the same data set as before, the overbounding standard deviations and mean values in the single and combined terms are given in Table 3. For the purpose of comparison, $\tilde{\sigma}_{\Delta \tilde{r} \Delta t_{\mathrm{G}}}$ and $\tilde{m}_{\Delta \tilde{r} \Delta t_{\mathrm{G}}}$ are given for the case when the correlations between $\Delta \tilde{r}$ and $\Delta t_{\mathrm{G}}$ are considered, i.e., for the term $\left(\Delta \tilde{r}-c \times \Delta t_{\mathrm{G}}\right)$. Outlier rejection was performed as in the previous section for the single and combined items of $\Delta \tilde{r}$ and $\Delta t_{\mathrm{G}}$. Epochs having valid values for all terms, i.e., the clocks, the radial orbits, and the combined clock and radial orbits, are used for the analysis.

From Table 3, it can be seen that $\tilde{\sigma}_{\Delta \tilde{r}}$ and $\tilde{m}_{\Delta \tilde{r}}$ in the worstcase are higher than the corresponding orbital contributions under the average-case (as listed in Table 2), i.e., the terms $\sqrt{\alpha_{\mathrm{R}}^{2} \times \tilde{\sigma}_{\mathrm{R}}^{2}+\alpha_{\mathrm{SW}}^{2} \times\left(\tilde{\sigma}_{\mathrm{S}}^{2}+\tilde{\sigma}_{\mathrm{W}}^{2}\right)} \quad$ and $\quad\left(\alpha_{\mathrm{R}} \times \tilde{m}_{\mathrm{R}}+\alpha_{\mathrm{SW}} \times\right.$ $\left.\left(\tilde{m}_{\mathrm{S}}+\tilde{m}_{\mathrm{W}}\right)\right)$. Likewise, the overbounding standard deviations and mean values of the combined orbital and clock errors $\tilde{\sigma}_{\Delta \tilde{r} \Delta t_{\mathrm{G}}}$ and $\tilde{m}_{\Delta \tilde{r} \Delta t_{\mathrm{G}}}$ are lower than those computed based on the single terms, i.e., the final $\tilde{\sigma}_{\mathrm{GNSS}}$ and $\tilde{m}_{\mathrm{GNSS}}$. Note that the slight differences in $\tilde{\sigma}_{\Delta t_{\mathrm{G}}}$ and $\tilde{m}_{\Delta t_{\mathrm{G}}}$ compared to those in the average-case are caused by the different epochs used in the analysis due to the outlier rejection of different parameters. Overall, the $\tilde{\sigma}_{\mathrm{GNSS}}$ and $\tilde{m}_{\mathrm{GNSS}}$ are higher than those in the average-case, i.e., around $0.1 \mathrm{~m}$ for $\tilde{\sigma}_{\mathrm{GNSS}}$ and sub-dm to dmlevel for $\tilde{m}_{\mathrm{GNSS}}$. The results are largely consistent with the User Range Accuracy (URA) analysis performed by Cheng et al. (2018) for the CNES (National Center for Space Studies) realtime products, stating that a URA of $0.15 \mathrm{~m}$ is a reasonable value for the GPS satellites. Note that the use of an overbounding mean value helps in reducing the overbounding standard deviation.

\section{Test results}

In this section, the orbital PEs and the PLs are processed for the LEO satellite GRACE FO-1 during the period August 14 to 19,2018 assuming a PMI of $10^{-5}$. The PMI of $10^{-5}$ is assumed to be uniformly distributed in the RSW directions, i.e., $\frac{1}{3} \times 10^{-5}$ in each direction, resulting in a $K_{q}$ value of 4.65. The orbits produced by JPL were used as the reference for the computation of the PEs. In Fig. 2, as an example, the absolute PEs and PLs are shown for the average-case projection using the MADOCA GPS products on August 14, 2018 with about 15-16 orbital cycles. The overbounding standard deviations $\left(\tilde{\sigma}_{\mathrm{GNSS}}\right)$ and mean values $\left(\tilde{m}_{\mathrm{GNSS}}\right)$ for the projected orbits and clocks were taken from Table 2. For the purpose of comparison, the cases considering and not considering the bias contribution $\left(\left(\left|S_{\mathrm{RSW}, q}\left(t_{i}\right)\right| \times \tilde{b}\right)\right.$ and $\left(\left|S_{\mathrm{RSW}, \mathrm{k}, q}\left(t_{i}\right)\right| \times \tilde{b}\right)$ in Section "Integrity monitoring") are both illustrated for the PLs in Fig. 2 as red and blue dots, respectively. Note that the bias contribution needs to be considered in IM, as without the mean value $\tilde{m}_{\mathrm{GNSS}}$, a much larger overbounding standard deviation $\tilde{\sigma}_{\text {GNSS }}$ could be needed to satisfy the overbounding characteristic of a Gaussian distribution. The blue dots in Fig. 2 are shown to illustrate the contribution of the bias term.

From Fig. 2, it can be seen that the PLs overbound the PEs in all three directions, and in both modes, for the test day. Due to the increased model strength, although having the same overbounding parameters, the PLs are much lower in the reduced-dynamic mode than in the kinematic mode. In addition, the highest PLs in the radial direction in the kinematic mode, caused by the high correlation between the radial component and the satellite clocks, do not appear to be higher than those in the other directions in the reduceddynamic mode due to de-correlation between the radial orbital component and the satellite clock parameter after applying the orbital dynamic models. Comparing Eq. (19) and Eq. (26), both the formal standard deviation $\tilde{\sigma}_{\mathrm{RSW}, q}$ and projection matrix $S_{\mathrm{RSW}, q}$ are increased in the kinematic mode due to the weaker model strength without using the dynamic model. More concretely, compared to the kinematic POD that solely relies on the GNSS observations, the 
Fig. 2 Absolute orbital PEs and PLs of the LEO satellite GRACE FO-1 (assumed to be the GNSS user) using the MADOCA GPS products in the kinematic mode (left) and the reduced-dynamic mode (right) on August 14, 2018
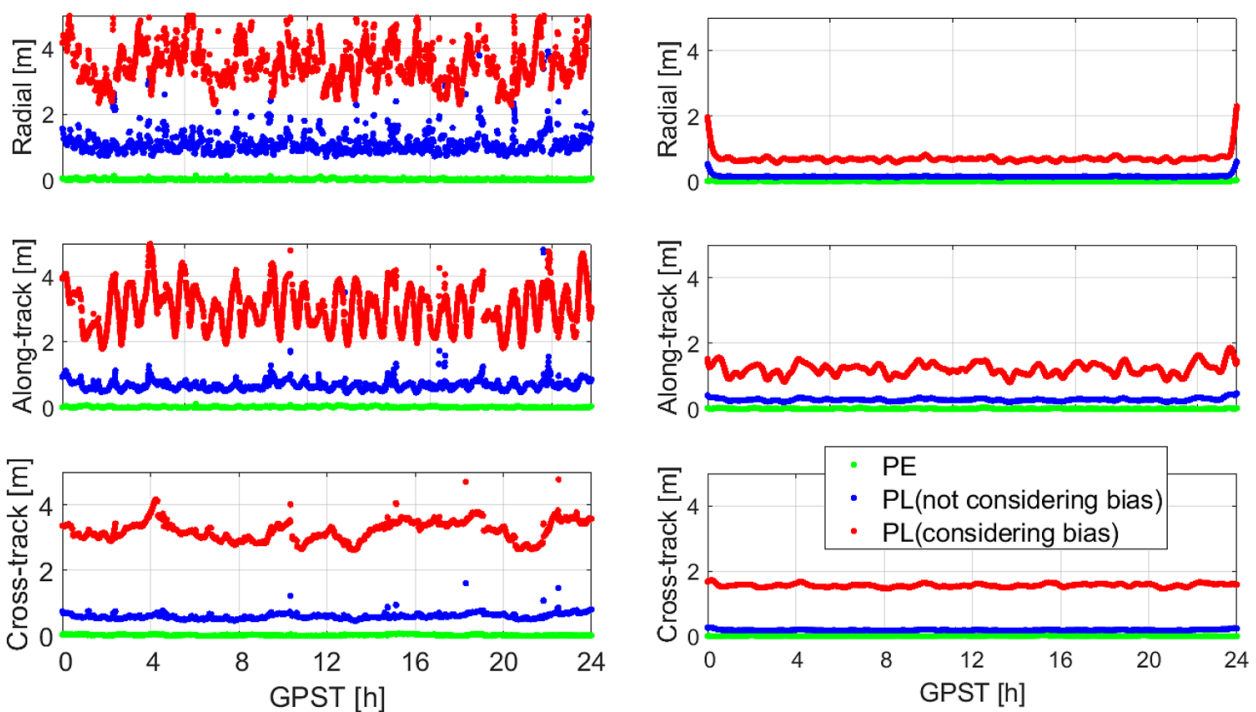

Fig. 3 Absolute PEs (left) and PLs assuming the average-condition applying the overbounding parameters of the MADOCA products (right). Note that the $y$-scales of the two sub-figures are different
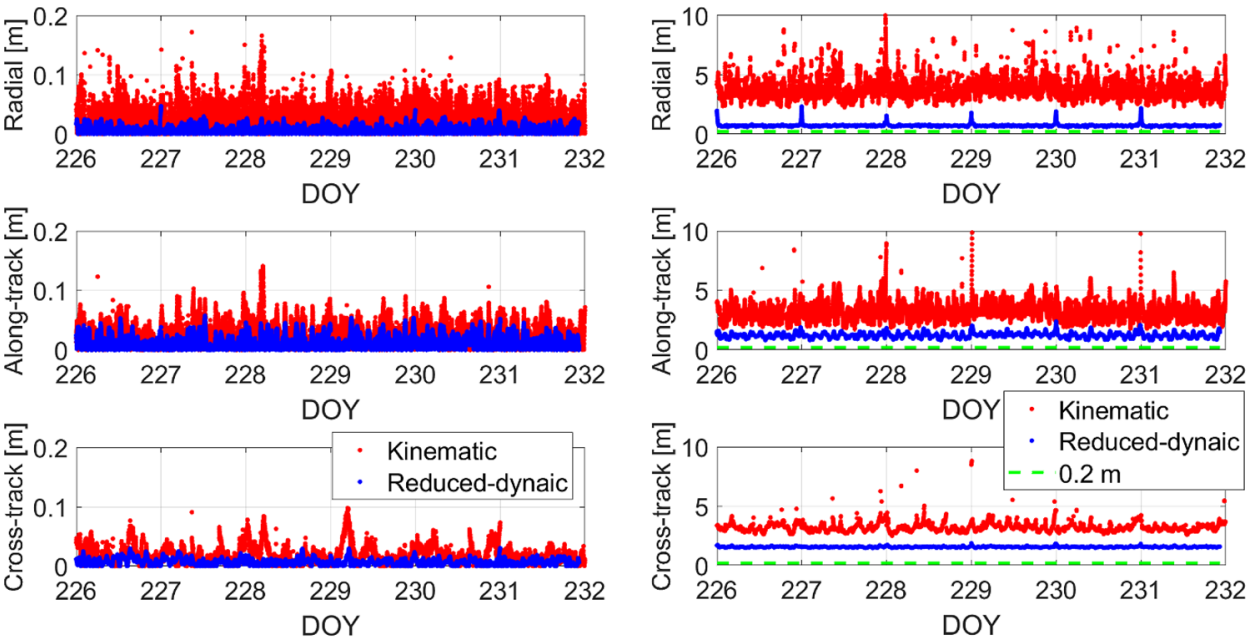

reduced-dynamic model gains more information from both the GNSS observations and the dynamic models.

Comparing the red and the blue dots in Fig. 2 i.e., with and without considering biases in the PLs, it can be seen that the bias contribution plays a significant role in the final PLs. This is partially caused by the fact that the absolute values of the projection matrices $S_{\mathrm{RSW}, q}\left(t_{i}\right)$ and $S_{\mathrm{RSW}, \mathrm{k}, q}\left(t_{i}\right)$ (see Section "Integrity Monitoring") were used in the IM, which pessimistically (as a conservative measure) assumes that the bias term always has the same sign as the corresponding values in the projection matrix.

Figure 3 shows the absolute PEs and the PLs for the average-condition applying the MADOCA overbounding parameters for all three directions. The LEO data of GRACE FO-1 from August 14 to 19,2018, were processed with the $\tilde{\sigma}_{\text {GNSS }}$ and the $\tilde{m}_{\mathrm{GNSS}}$ taken from Tables 2 and 3 for the averagecase and the MADOCA products. It can be observed that the reduced-dynamic mode has significantly reduced both of the
PEs and the PLs compared to the kinematic mode. The figure shows that the PLs bound all PEs in the three directions and during the entire test period (see the $0.2 \mathrm{~m}$ cap in the right panel). Table 4 lists the mean value of the absolute PEs and the PLs, and the corresponding IM availabilities with an assumed AL of $4 \mathrm{~m}$. This is an example presented for demonstration purposes, showing the availability differences for different estimation modes and under the average-case and the worst-case projections. The availability is defined as the ratio when PL is smaller than $\mathrm{AL}$ in the corresponding direction. The values in Table 4 distinguish between the average-case and the worst-case.

While the PLs in the average-case projection can indeed bound all the PEs in the three directions and during the entire test period, the worst-case projection provides safer but overall pessimistic PLs. Benefiting from the increased model strength, as shown in Table 4, applying the dynamic model can effectively reduce the mean PLs from about 
Table 4 Statistics of the orbital positioning errors (PEs) and protection levels (PLs) of GRACE FO-1 for the period August 14 to 19, 2018. The values separated by " " " are given in the radial, along-track and cross-track directions, respectively

\begin{tabular}{|c|c|c|c|c|c|}
\hline \multirow[t]{2}{*}{ Estimation mode } & \multirow[t]{2}{*}{ Mean abs. PE [m] } & \multicolumn{2}{|l|}{ Mean PL [m] } & \multicolumn{2}{|l|}{ Availability [\%] } \\
\hline & & Average-case & Worst-case & Average-case & Worst-case \\
\hline \multicolumn{6}{|l|}{ MADOCA } \\
\hline Kinematic & $0.028 / 0.026 / 0.019$ & $3.83 / 3.25 / 3.20$ & $7.24 / 6.39 / 6.36$ & $68.0 / 85.4 / 97.9$ & $0.0 / 3.2 / 0.0$ \\
\hline Reduced-dynamic & $0.009 / 0.014 / 0.007$ & $0.70 / 1.23 / 1.56$ & $1.38 / 2.42 / 3.20$ & $100 / 100 / 100$ & 99.95/99.97/100 \\
\hline \multicolumn{6}{|l|}{ IGS RTS } \\
\hline Kinematic & $0.049 / 0.045 / 0.036$ & $4.50 / 3.74 / 3.55$ & $6.45 / 5.52 / 5.30$ & $42.6 / 66.8 / 82.4$ & 2.3/16.6/0.6 \\
\hline Reduced-dynamic & $0.011 / 0.022 / 0.014$ & $0.72 / 1.27 / 1.55$ & $1.06 / 1.87 / 2.39$ & 100/100/100 & $100 / 100 / 100$ \\
\hline
\end{tabular}

3-4 $\mathrm{m}$ in the kinematic mode to around $1 \mathrm{~m}$ in the reduceddynamic mode for all three directions for the average-case assumption. Under such conditions, the availabilities reach $100 \%$ for an AL of $4 \mathrm{~m}$ as shown in Table 4, and are above 99\% even with the AL decreased to $2 \mathrm{~m}$.

\section{PLs with different overbounding parameters}

To test the impact of different overbounding parameters, the PLs were computed using the satellite geometry provided by the GRACE FO- 1 and the MADOCA products on August 14,2018 , with the value of $\tilde{\sigma}_{\text {GNSS }}$ varying from 0.05 to $0.15 \mathrm{~m}$ and the value of $\tilde{m}_{\mathrm{GNSS}}$ varying from 0 to $0.15 \mathrm{~m}$. Figure 4 shows the change of the mean PLs with respect to the $\tilde{m}_{\mathrm{GNSS}}$ in the kinematic (top) and reduced-dynamic modes (bottom).

As shown in Fig. 4, compared to the $\tilde{\sigma}_{\mathrm{GNSS}}$, the mean PLs are more influenced by the $\tilde{m}_{\mathrm{GNSS}}$ of the projected GNSS orbital and clock errors. The results for LEO POD motivate investigations into further reducing the overbounding mean values. With $\tilde{m}_{\mathrm{GNSS}}$ within $5 \mathrm{~cm}$, the mean values of PLs in the reduced-dynamic mode are within $2 \mathrm{~m}$ for the averagecase even with a large $\tilde{\sigma}_{\mathrm{GNSS}}$ up to $0.15 \mathrm{~m}$. With $\tilde{m}_{\mathrm{GNSS}}$ within $2 \mathrm{~cm}$, the mean values of PLs are limited to $1 \mathrm{~m}$ under the same conditions.

\section{PLs with different duty cycles}

For mini-satellites such as CubeSats, the limited onboard power might not allow for GNSS observations to be continuously collected over an entire period, as power might need to be used for tasks other than positioning. As such, duty cycles smaller than $100 \%$ are often applied for the GNSS tracking (Lantto and Gross 2018). The reduced data decreases the model strength and the precision of the estimated orbital parameters. However, in the reduced-dynamic mode the orbital positions and PLs can still be computed over the entire period, benefiting from the dynamic model and numerical integration (Wang et al. 2020).

The mean PLs are computed and shown in Fig. 5 for duty cycles varying from 40 to $100 \%$ in the reduced-dynamic mode. The geometry of GRACE FO-1 on August 14, 2018, was used for the tests, and the overbounding parameters of the MADOCA products in the average-case (Table 2) were applied for the analysis.

From Fig. 5 it can be seen that the mean value of PLs increase, i.e., tending to reduced availability, in all three directions with decreasing duty cycles. This is due to the reduced model strength when decreasing the amount of data used for POD. However, the mean PLs after the increase are limited to about $2 \mathrm{~m}$ even when reducing the duty cycle to $40 \%$. With an AL set to $4 \mathrm{~m}$, the availabilities are still above $98 \%$ when the duty cycle is reduced to $40 \%$.

\section{Concluding remarks}

Integrity monitoring (IM) is an essential task in guaranteeing the safety and reliability of a positioning service. This study proposed new IM strategies for GNSS-based near-realtime LEO POD, investigating how different error sources and model strength could influence their final PLs.

As a condition for high accuracy near-real-time LEO POD, the overbounding standard deviation and mean value were studied for the SISRE of real-time GPS orbits and clocks using the IGS RTS and MADOCA products. The investigation distinguished between the average-case and worst-case projections. Using the geometry of the LEO satellite GRACE FO-1, the results showed that for the averagecase assumption, the overbounding standard deviations are at the sub-dm to dm-level, and the overbounding mean values are within $5 \mathrm{~cm}$, while in the worst-case the overbounding standard deviations are about $0.1 \mathrm{~m}$ and the corresponding mean values are at the sub-dm to dm-level.

The PLs were computed using both the real-time GNSS products and real data for the LEO satellite GRACE FO-1. Assuming a PMI of $10^{-5}$, the strong dynamic models and the improved model strength bring the mean PLs from about 3-4 $\mathrm{m}$ in the kinematic mode down to about $1 \mathrm{~m}$ in the reduced-dynamic mode for the average-case assumption in the radial, along-track and cross-track directions, where the PL safely bound all the orbital PEs. The worstcase projection leads to safer, but overall more conservative (pessimistic) PLs, having mean values of about $1-3 \mathrm{~m}$ in 
Fig. 4 Mean PLs for the average-case assumption in the (left) radial, (middle) along-track and (right) cross-track directions. The top and bottom panels correspond to the kinematic and reduced-dynamic modes, respectively
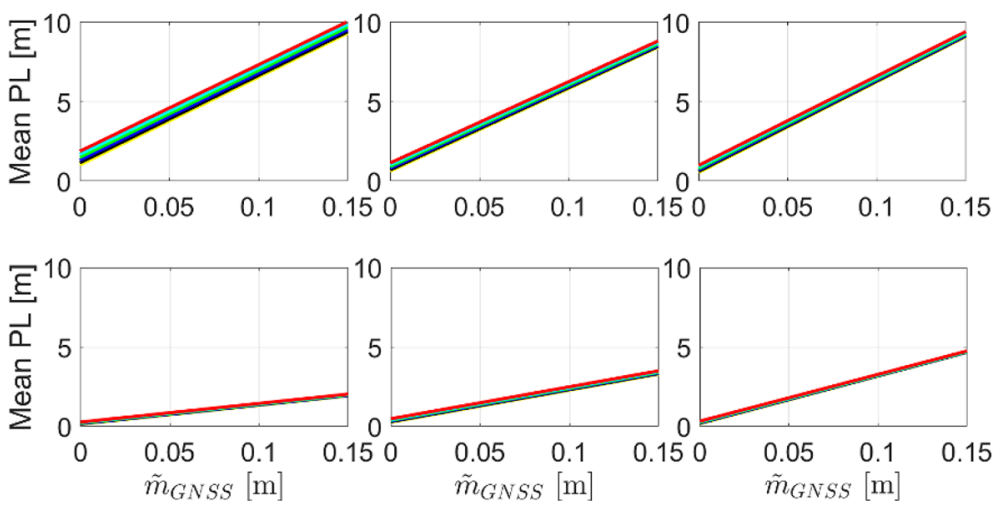

$\tilde{\sigma}_{G N S S}=0.05 \mathrm{~m}$

$-\tilde{\sigma}_{G N S S}=0.07 \mathrm{~m}$

$-\tilde{\sigma}_{G N S S}=0.09 \mathrm{~m}$

$-\tilde{\sigma}_{G N S S}=0.11 \mathrm{~m}$

$-\tilde{\sigma}_{G N S S}=0.13 \mathrm{~m}$

$-\tilde{\sigma}_{G N S S}=0.15 \mathrm{~m}$

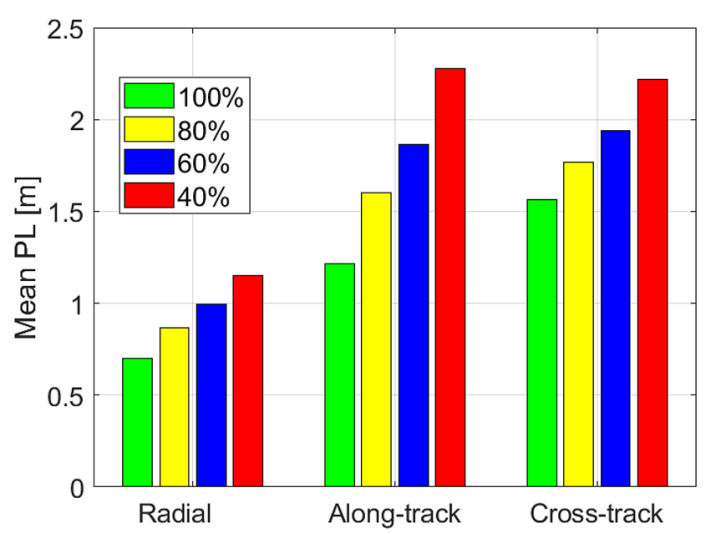

Fig. 5 Mean PLs with different duty cycles in the reduced-dynamic mode applying the overbounding parameters using MADOCA products for the average-case assumption

the reduced-dynamic mode and over $5 \mathrm{~m}$ in the kinematic mode. The IM availabilities in the reduced-dynamic mode and for the average-case projection are above $99 \%$ in all three directions when the AL is set to $2 \mathrm{~m}$. The analysis further showed that the overbounding mean value of the SISRE plays a determining role in the final PLs. For small satellites, with duty cycles down to $40 \%$, the mean values of PLs (in the reduced-dynamic mode and the average-case projection) increase compared to those with a $100 \%$ duty cycle, but are limited to about $2 \mathrm{~m}$ using the overbounding parameters of the MADOCA products. The corresponding IM availabilities are shown to be above $98 \%$ when the AL is set to $4 \mathrm{~m}$, even with the duty cycle reduced to $40 \%$.

Acknowledgements The work is funded by the Australian Research Council Discovery Project: Tracking Formation-Flying of Nanosatellites Using Inter-Satellite Links, Project Number (DP 190102444), and the Chinese Academy of Sciences (CAS) "Light of West China" Program (No. XAB2018YDYL01).
Funding Australian Research Council, DP 190102444, and Chinese Academy of Sciences, "Light of West China" Program, No. XAB2018YDYL01.

Data availability The MADOCA products were obtained from the JAXA ftp://mgmds01.tksc.jaxa.jp/mdc1/. The IGS RTS products were obtained from the NASA CDDIS https://cddis.nasa.gov/archive/ gnss/products/rtpp/. The data of GRACE FO-1 were obtained from the NASA JPL https://podaac-tools.jpl.nasa.gov/drive/files/allData/grace fo/L1B/JPL/RL04/ASCII/.

Open Access This article is licensed under a Creative Commons Attribution 4.0 International License, which permits use, sharing, adaptation, distribution and reproduction in any medium or format, as long as you give appropriate credit to the original author(s) and the source, provide a link to the Creative Commons licence, and indicate if changes were made. The images or other third party material in this article are included in the article's Creative Commons licence, unless indicated otherwise in a credit line to the material. If material is not included in the article's Creative Commons licence and your intended use is not permitted by statutory regulation or exceeds the permitted use, you will need to obtain permission directly from the copyright holder. To view a copy of this licence, visit http://creativecommons.org/licenses/by/4.0/.

\section{References}

Allahvirdi-Zadeh A, Wang K, El-Mowafy A (2021) POD of small LEO satellites based on precise real-time MADOCA and SBASaided PPP corrections. GPS Solut 25:31. https://doi.org/10.1007/ s10291-020-01078-8

Blanch J, Walter T, Enge P, Lee Y, Pervan B, Rippl M, Spletter A (2012) Advanced RAIM user algorithm description: Integrity support message processing, fault detection, exclusion, and protection level calculation. In: Proceedings of ION GNSS, Institute of Navigation, Nashville, TN, September 2012, pp 2828-2849

Blanch J, Walter T, Enge P (2018) Gaussian bounds of sample distributions for integrity analysis. IEEE Trans Aerosp Electron Syst 55(4):1806-1815. https://doi.org/10.1109/TAES.2018.2876583

Cheng C, Zhao Y, Li L, Cheng J, Sun X (2018) Preliminary analysis of URA characterization for GPS real-time precise orbit and clock products. In: Proceedings of the 2018 IEEE/ION position, location and navigation symposium (PLANS), Monterey, CA, USA, 23-26 April 2018. https://doi.org/10.1109/PLANS.2018.8373434 
Dach R, Lutz S, Walser P, Fridez P (2015) Bernese GNSS software version 5.2. University of Bern, Bern Open Publishing, Bern, Switzerland. https://doi.org/10.7892/boris.72297

El-Mowafy A, Kubo N (2018) Integrity monitoring for positioning of intelligent transport systems using integrated RTK-GNSS, IMU and vehicle odometer. IET Intel Transport Syst 12(8):901-908. https://doi.org/10.1049/iet-its.2018.0106

El-Mowafy A, Deo M, Kubo N (2017) Maintaining real-time precise point positioning during outages of orbit and clock corrections. GPS Solut 21:937-947. https://doi.org/10.1007/ s10291-016-0583-4

EUROCAE (2019) Minimum operational performance standard for Galileo/global positioning system/satellite-based augmentation system airborne equipment. The European organisation for civil aviation equipment, ED-259, February 2019

Ge H, Li B, Ge M, Zang N, Nie L, Shen Y, Schuh H (2018) Initial assessment of precise point positioning with LEO enhanced global navigation satellite systems (LeGNSS). Remote Sens 10(7):984. https://doi.org/10.3390/rs10070984

Gu D, Ju B, Liu J, Tu J (2017) Enhanced GPS-based GRACE baseline determination by using a new strategy for ambiguity resolution and relative phase center variation corrections. Acta Astronaut 138:176-184. https://doi.org/10.1016/j.actaastro.2017.05.022

Hassan T, El-Mowafy A, Wang K (2020) A review of system integration and current integrity monitoring methods for positioning in intelligent transport systems. IET Intel Transport Syst 15(1):4340. https://doi.org/10.1049/itr2.12003

Heng L, Gao GX, Walter T, Enge P (2011) Statistical characterization of GPS signal-in-space errors. In: Proceedings of 2011 ION ITM, San Diego, CA, USA, 24-26 January 2011, pp 312-319

Hauschild A, Tegedor J, Montenbruck O, Visser H, Markgraf M (2016) Precise onboard orbit determination for LEO satellites with realtime orbit and clock corrections. In: Proceedings of ION GNSS+ 2016, Institute of Navigation, Portland, Oregon, USA, September 12-16, 2016, pp 3715-3723

Han Y, Wang L, Fu W, Zhou H, Li T, Xu B, Chen R (2020) LEO navigation augmentation constellation design with the multi-objective optimization approaches. Chin J Aeronaut 34(4):265-278. https:// doi.org/10.1016/j.cja.2020.09.005

Lantto S, Gross JN (2018) Precise orbit determination using duty cycled GPS observations. In: Proceedings of the 2018 AIAA modeling and simulation technologies conference, AIAA SciTech Forum, Kissimmee, FL, USA, 8-12 January 2018; AIAA: Reston, VA, USA, 2018. https://doi.org/10.2514/6.2018-1393

Lyard F, Lefevre F, Letellier T, Francis O (2006) Modelling the global ocean tides: modern insights from FES2004. Ocean Dyn 56:394415. https://doi.org/10.1007/s10236-006-0086-x

Montenbruck O (2017) Space Applications. In: Teunissen PJG, Montenbruck $\mathrm{O}$ (eds) Springer handbook of global navigation satellite systems. Springer International Publishing, Cham, Switzerland, pp 933-964

Montenbruck O, Gill E (2000) Satellite orbits: models, methods and applications. Springer, Berlin, Heidelberg, Germany. https://doi. org/10.1007/978-3-642-58351-3

Montenbruck O, Gill E, Kroes R (2005) Rapid orbit determination of LEO satellites using IGS clock and ephemeris products. GPS Solut 9(3):226-235. https://doi.org/10.1007/s10291-005-0131-0

Montenbruck O, Hauschild A, Andres Y, von Engeln A, Marquardt C (2013) (Near-) real-time orbit determination for GNSS radio occultation processing. GPS Solut 17:199-209. https://doi.org/ 10.1007/s10291-012-0271-y

Montenbruck O, Steigenberger P, Hauschild A (2015) Broadcast versus precise ephemerides: a multi-GNSS perspective. GPS Solut 19:321-333. https://doi.org/10.1007/s10291-014-0390-8
Petit G, Luzum B (2010) IERS Conventions. IERS Technical Note, 36. Verlag des Bundesamtes für Kartographie und geodäsie, frankfurt am main, Germany, 2010. 179p, ISBN 3-89888-989-6

Pavlis NK, Holmes SA, Kenyon SC, Factor JK (2008) An Earth gravitational model to degree 2160: EGM2008. European geosciences union general assembly, Vienna, Austria, 13-18 April 2008

Reid TGR, Neish AM, Walter T, Enge PK (2018) Broadband LEO constellations for navigation. Navigation 65(2):205-220. https:// doi.org/10.1002/navi.234

Standish EM (1998) The JPL planetary and lunar ephemerides, DE405/ LE405. JPL IOM 312, F-98-048

Shively CA (2014) Analysis of probability of misleading information for LAAS signal in space. Navig J Instit Navig 47(2):128-142. https://doi.org/10.1002/j.2161-4296.2000.tb00208.x

Wang K, Allahvirdi-Zadeh A, El-Mowafy A, Gross JN (2020) A sensitivity study of POD using dual-frequency GPS for cubesats data limitation and resources. Remote Sens 12(13):2107. https://doi. org/10.3390/rs12132107

Wang K, El-Mowafy A, Rizos C, Wang J (2021) SBAS DFMC service for road transport: positioning and integrity monitoring with a new weighting model. J Geodesy 95(3):29. https://doi.org/10. 1007/s00190-021-01474-z

Wen HY, Kruizinga G, Paik M, Landerer F, Bertiger W, Sakumura C, Bandikova T, Mccullough C (2019) Gravity recovery and climate experiment follow-On (GRACE-FO). Level-1 data product user handbook vol JPL D-56935 (URS270772)

Wu J, Wang K, El-Mowafy A (2020) Preliminary performance analysis of a prototype DFMC SBAS service over Australia and AsiaPacific. Adv Space Res 66(6):1329-1341. https://doi.org/10. 1016/j.asr.2020.05.026

Rife J, Pullen S, Enge P, Pervan B (2006) Paired overbounding for nonideal LAAS and WAAS error distributions. IEEE Trans Aerosp Electron Syst 42(4):1386-1395. https://doi.org/10.1109/TAES. 2006.314579

Zhang S, Du S, Li W, Wang G (2019) Evaluation of the GPS precise orbit and clock corrections from MADOCA real-time products. Sensors 19(11):2580. https://doi.org/10.3390/s19112580

Publisher's Note Springer Nature remains neutral with regard to jurisdictional claims in published maps and institutional affiliations.

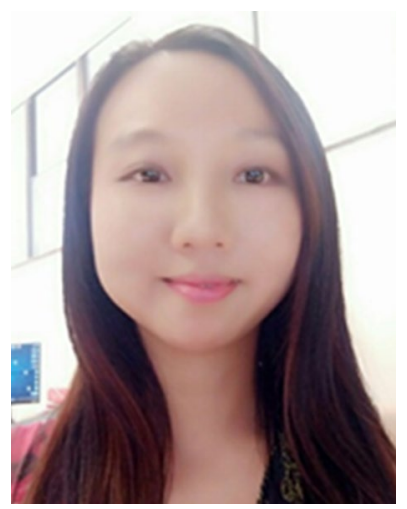

Kan Wang is an Associate Professor at the National Time Service Center, Chinese Academy of Sciences. She received her Ph.D. in GNSS advanced modeling from ETH Zurich in 2016 and worked at Curtin University until 2021. Her research interests include high-precision GNSS positioning, LEO precise orbit determination, SBAS and integrity monitoring. 


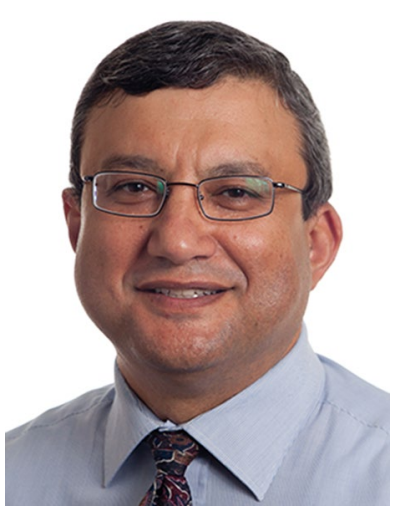

Ahmed El-Mowafy obtained his Ph.D. from the University of Calgary, Canada, in 1995 and is a Professor of Positioning and Navigation and Director of Graduate Research, School of Earth and Planetary Sciences, Curtin University, Australia. He has 190 publications in precise positioning and navigation using GNSS, quality control, integrity monitoring and estimation theory.

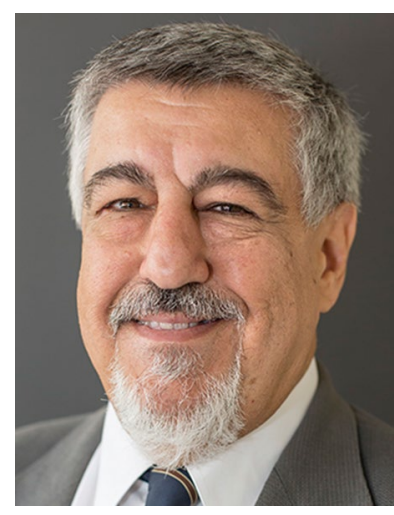

Chris Rizos is an Emeritus Professor, School of Civil \& Environmental Engineering, UNSW. Chris is past president of the International Association of Geodesy (IAG), a former member of the Executive and Governing Board of the International GNSS Service (IGS), and is currently president-elect of the International Union of Geodesy $\&$ Geophysics. Chris has been researching the technology and applications of GPS since 1985 and is an author/co-author of over 650 journal and conference

papers. 